\title{
Anesthetic Experience of Patient with Lambl's Excrescences
}

\author{
Nam Yung Kim ${ }^{1}$, Seongsik Kang ${ }^{2}$, Se Jin $\mathrm{Kim}^{3}$, So Yeon $\mathrm{Cho}^{4}$ \\ ${ }^{1}$ Department of Anesthesiology and Pain Medicine, Institute of Medical Sciences, Kangwon National University Hospital, School of \\ Medicine, Chuncheon, South Korea \\ Email: namyung 15 [AT] naver.com \\ ${ }^{2}$ Department of Anesthesiology and Pain Medicine, Institute of Medical Sciences, Kangwon National University Hospital, School of \\ Medicine, Chuncheon, South Korea \\ Email: sskang0504 [AT] gmail.com \\ ${ }^{3}$ Department of Anesthesiology and Pain Medicine, Institute of Medical Sciences, Kangwon National University Hospital, School of \\ Medicine, Chuncheon, South Korea \\ Email: ksj8826 [AT] naver.com \\ ${ }^{4}$ Department of Anesthesiology and Pain Medicine, Institute of Medical Sciences, Kangwon National University Hospital, School of \\ Medicine, Chuncheon, South Korea \\ Email: ju_nu7 [AT] naver.com
}

\begin{abstract}
Lambl's excrescences $(L E)$ are mobile, thin, filiform structures that occur at sites of valve closure. $L E$ are mostly asymptomatic but atheroma from LE may embolize to cerebrovascular arterial territory causing stroke. A 79 year old man with mechanical ileus of small bowel loop and pelvic mass scheduled for palliative enteroenterostomy. His transthoracic echocardiography showed a filiform hyperechoic mass attached to the tip of noncoronary cusp, suggestive of LE. To prevent cardioembolic stroke during perioperative period, we tried to prevent blood pressure fluctuation during surgery. Also we monitored regional cerebral oxygen saturation by cerebral/somatic oximeter for early detection of cerebral ischemia. A patient had no neurological changes and signs of cerebral infarction after surgery.
\end{abstract}

Keywords--- Lambl's excrescences, embolism, stroke, echocardiography

\section{INTRODUCTION}

Lambl's Excrescences first described in 1856, by Vilém Dušan Lambl, a Bohemian physicianare.[1] LEare mobile, thin, filiform strands that found on atrial surface of the mitral valve, or ventricular surface of the aortic valve. Aortic and mit ral valves are the most common locations of LE. However, they occur anywhere on the valve [2]. MostLE are as ymptomatic, therefore LE diagnosed by cardiac echocardiography (transesophageal echocardiogram or tran sthoracic echocardiogram) incidentally [3]. LE are seen increasingly with advancing age and improved image quality. LEconsidered normal variants that represent a form of degenerative change of the valve[4]. However atheroma from LE have a potential risk of embolizing to distant organs causing stroke[5]. In this care report, we describe patient with LE who was scheduled for palliative enteroenterostomy and dis cussion with anesthetic management of LE.

\section{PRESENTATION OF CASE}

The patient(height: $167 \mathrm{~cm}$, body weight: $50.6 \mathrm{~kg}$ ) was a 79 yearold man with atrial fibrillation without anticoagulation, and the past history of lower anterior resection with incidental appendectomy due to rectal cancer 10 years ago. he was diagnosed with prostate cancer in January 2018 and received hormone therapy until august 2018. He vis ited the emergency department with poor oral intake and general weakness on January 2019. The abdomen and pelvis computed tomography scan indicated mechanical ileus of small bowel loop and increased size of pelvic mass.

The patient scheduled for palliative enteroenterostomy and underwent a series of preoperative evaluation including transthoracic echocardiography. Laboratory value were within normal range. Chest radiography showed multiple metas tatic nodules and confluent emphysema in the both lungs. Electrocardiogram showed atrial fibrillation and vital signs were within normal limit except heart rate $120 \mathrm{beats} / \mathrm{min}$. He didn't complain any cerebrovascular accident symptoms and his neurologic examyielded normal results. 
Transthoracic echocardiography showed a $5.4 \mathrm{~mm}$ sized filiform hyperechoic mass attached to the tip of noncoronary cusp, suggestive of LE(Figure 1). There was no regional wall motion abnormality and LV ejection fraction was $64 \%$. Further findings were both atrial enlargement, moderate mitral regurgitation, moderate tricuspid regurgitation and moderate pulmonary hypertension.

Figure 1: Trans thoracic echo showing Lambl's excrecences (Arrow)

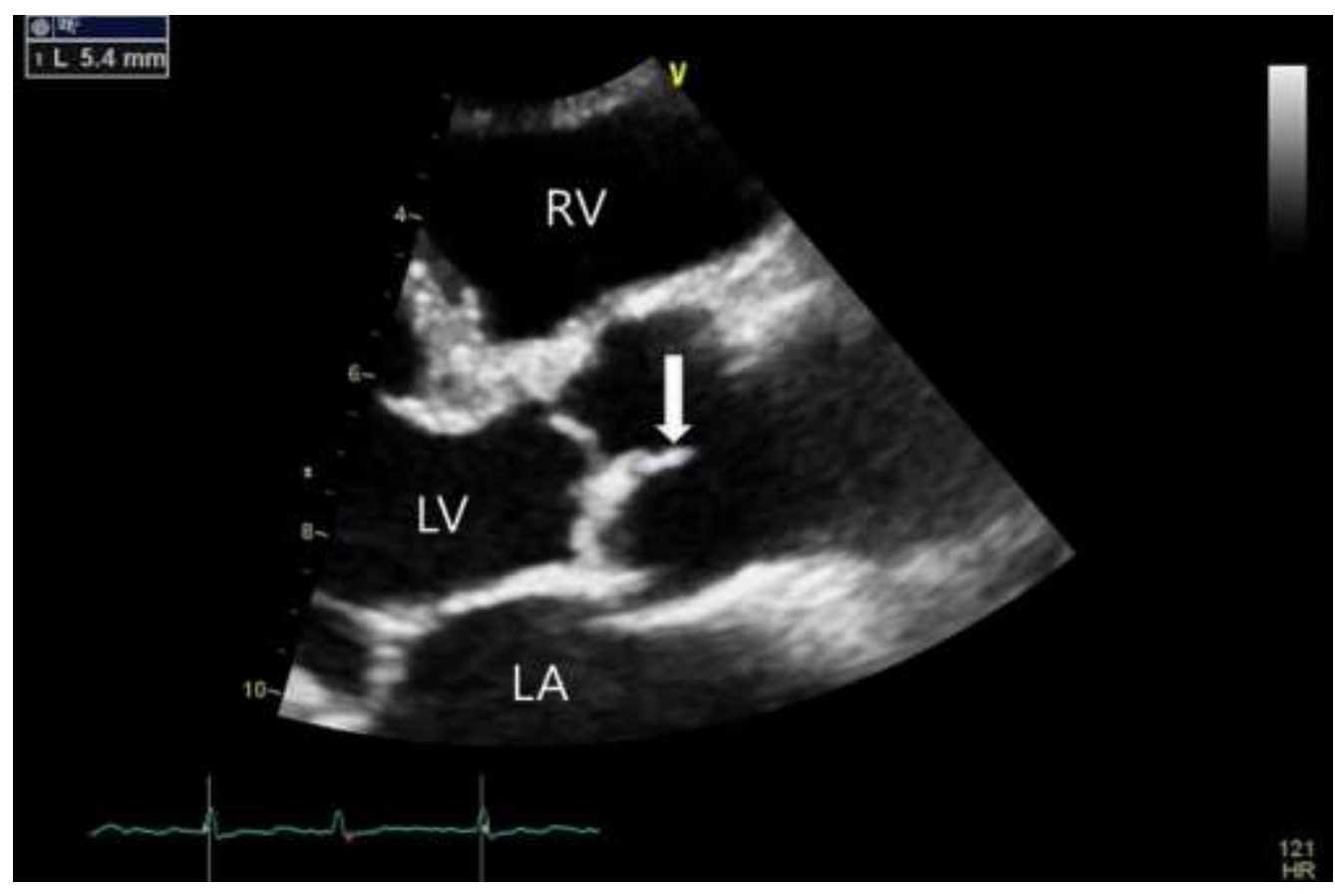

In the operating room, intraoperative monitoring was started using electrocardiogram, non-invasive blood pressure, pulse oximetry and cerebral oximeter. Anes thesia was induced with intravenous fentanyl $50 \mathrm{mcg}, 2 \%$ lidocaine $20 \mathrm{mg}$, propofol $80 \mathrm{mg}$, rocuronium $40 \mathrm{mg}$. After endotracheal intubation, sevoflurane was used for anesthetic maintenance. The left radial artery, left external jugular vein were cannulated. Blood pressure was maintained within the range of systolic 90-130 $\mathrm{mmHg} /$ diastolic $60-80 \mathrm{mmHg}$ and heart rate was maintained within $90-130$ beats/min. To stabilize hemodynamics of patient, we injected phenylephrine and esmolol intermittently. There was no significant change in the regional cerebral oxygen saturation during operation.

The operating time was 85 minutes and there were no complications. sugammadex $200 \mathrm{mg}$ was administered intravenously for reversal of muscle relaxation. The patient was extubated uneventfully and transfered to the recovery room. While in the recovery room, the patient was hemodymically stable, his mental status was back to baseline.

After postoperative 2 days, he was started on dual anti-platelet therapy to prevent thromboemolis $m$ after cardiology consultation.

\section{DISCUSSION}

LE considered a rare cause of cardioembolic stroke, an atheroma from LE or LE fragments may embolize to cerebrovascular arterial territory causing stroke. It is rare for an anes thesiologist to meet a LE patient. Most patients with $\mathrm{LE}$ are as ymptomatic, found LE during preoperative examinations incidentally.

Because there is no consensus on the treatment of LE, the management is largely based upon case reports in the literature. The general therapeutic approach to LE with a single embolic ischemic event is either anticoagulation or anti-platelet therapy[6]. In as ymptomatic patients, observation is reasonable, but some physicians prefer antiplatelet therapy. If no other embolic sources are found after the first stroke, some authors recommend anticoagulation or antiplatelet therapy. Surgical intervention should be performin patients who experience recurrent stroke in the absence of an obvious cause and in patients whose LE causes a functional abnormality, such as coronary occlusion that leads to myocardial is chemia or valve obstruction[7].

In accordance with this recommendation, although our patient has nothad cerebral infarction during the past year, since he had atrial fibrillation on the electrocardiogram, we decided to start dual antiplatelet therapy postoperatively. Also there are no definitive guidelines for the anesthetic management of patients with LE. In this case, to prevent and detect earty cardioembolic stroke during perioperative period, we carefully monitored blood pressure, cerebral oxygen saturation and 
neurologic status of patient. Intraoperative period can be critical time for LE patient because blood pres sure changes frequently and physician can not monitor neurological status.

Anes thesiologist should recognize that LE may caus e cardioembolic stroke during perioperative period. We recommend that maintain a stable blood pressure and heart rate and avoiding sudden fluctuation during intraoperative period. Maintaining adequate cerebral perfusion for LE patient also important. Monitoring regional cerebral oxygen s aturation by us ing cerebral oximeter might provide information of cerebral perfu sion status and help to detect cerebral ischemia early. Intraoperative use of transesophageal echocardiography can be useful because we can monitor valvular structure and function. However we did not use the transesophageal echocardiography because the patient has no cerebral infarction in the past and resorce limitation.

\section{CONCLUSION}

$\mathrm{LE}$ is a rare cause of cardioembolic stroke. Although there is no consensus on the anesthetic managent of LE patients, anes thesiologist should be carefulattention to prevention and early of thromboembolis mduring surgery.

\section{REFERENCES}

1. Lambl VA. Papillare excrescenzen und der semilunar-klappe der aorta. Wien Med Wochenschr. 1856; 16 :244247.

2. Aziz F, Baciewicz FA. Lambl's excrescences: review and recommendations. TexHeart Inst J, 2007; 34: 366-68

3. Voros S, Nanda NC, Thakur AC et al: Lambl's excrescences (valvular strands). Echocardiography, 1999; 16: $399-414$

4. Amstrong W, Ryan T. Feigenbaum's Echocardiography 7th ed. Lippincott Williams \& Wilkins. 2010; 11: 293

5. Chu A, Aung TT, Sahalon H, Choksi V, Feiz H. Lambl's Excrescence As sociated with Cryptogenic Stroke: A CaseReport and Literature Review. AmJ Case Rep. 2015; 16:876-881.

6. Kamran H, Patel N, Singh G, Pasricha V, Salifu M, et al. (2016) Lambl's excrescences: A case report and review of the literature. Clinical case reports and reviews 2: 486.

7. Davogustto G, Fernando RR, Loghin C (2015) Lambl's excrescence, migrainous headaches, and "tiger stripes": puzzling findings in one patient. TexHeart Inst J 42: 70-72. 